\title{
Isolation of Microalgae for Biomass and Lipid Enhancement through Plackett-Burmann Design
}

\author{
Premjyoti C. Patil ${ }^{*}$ and Bharati S. Meti \\ Department of Biotechnology, Basaveshwar Engineering College, Bagalkot, \\ Karnataka-587102, India \\ *Corresponding author
}

\begin{tabular}{|l|}
\hline Ke y w or d s \\
$\begin{array}{l}\text { Chlorella, 18s rRNA, } \\
\text { optimization, Plackett- } \\
\text { Burman, Biomass, Algal } \\
\text { lipid }\end{array}$ \\
\hline Article Info \\
\hline $\begin{array}{l}\text { Accepted: } \\
\text { 20 February } 2018 \\
\text { Available Online: } \\
\text { 10 March } 2018\end{array}$ \\
\hline
\end{tabular}

\section{Introduction}

Biodiesel is currently receiving much attention due to its potential as a sustainable and environment friendly alternative fuel. Use of edible food crops for the production of biodiesel has raised the concerns of food insecurity. Therefore, algae as a feedstock for the production of biofuels has emerged at the fore front in the biofuel research (Chisti 2007, Chisti, 2008; Sivasubramanian, 2009, Michael et al., 2010, Wayer et al., 2010 John et al., 2011). Microalgae are source of all nutrients
The algae are one among the biomass energy source which can be promising. The microalgae are the diverse organisms which can inhabit in different climatic conditions and adapt themselves and perpetuate forming their own niche. There is need to improve the local flora for biomass and lipid content instead of culturing exotic species. Chlorella the green microalgae, was isolated from fresh water and was characterized through molecular studies using 18s rRNA homology. The study has revealed that isolated species belong to Chlorophytes (green microalgae). The isolated species was further subjected to media optimization studies through Plackett-Burman which has revealed Urea being the highest positive effect of (7.6833) among seven variables $\left(\mathrm{NaNO}_{3}, \mathrm{CaCl}_{2}, \mathrm{MgSO}_{4}\right.$, $\mathrm{K}_{2} \mathrm{HPO}_{4}, \mathrm{KH}_{2} \mathrm{PO}_{4}$, Urea, 2-4,D) $\mathrm{R}^{2}$ value of $93.16 \%$ and $\mathrm{p}$ value of $0.033($ at $5 \%$ ) and $\mathrm{K}_{2} \mathrm{HPO}_{4}$ had a negative effect (-0.3833) for Biomass. $\mathrm{NaNO}_{3}$ had highest positive effect and $\mathrm{K}_{2} \mathrm{HPO}_{4}$ had least effect on the lipid content and $\mathrm{R}^{2}$ value of $61.14 \%$ and $\mathrm{p}$ value of 0.5788 (at $5 \%$ ) indicating the variables had less influence compared to Biomass. Further the variables which had highest positive effect on biomass and lipid will be selected for Response Surface methodology studies, aim will be to enhance both components in a single media, which will be the future scope of work. 
competition with food and land resources. In order to combat with the problem, algae can be better alternate source for biomass energy as the algae grows in wide conditions right from fresh water to waste water. They have greater adaptability for different salinity and $\mathrm{pH}$ conditions. Algae can be used to treat the waste water and same biomass can be harvested for biomass. The obtained biomass can be used to extract lipid and can be extracted for biodiesel and leftover biomass can be fermented for ethanol production or else can be explored for other purposes like proteins for feed and micronutrients for other needs. But the level of toxicity has to be evaluated. The laboratory studies need to begin with collection, isolation and characterization and later on strain improvement. In the current study an attempt has been made to collect, isolate and characterize the isolated species. Among different isolates Chlorella sp. axenic culture was obtained, which was supported by partial sequencing of $18 \mathrm{~S}$ rRNA homology study and phylogenetic analysis. The nucleotide partial sequence of isolate Chlorella sorokiniana PCPBEC was submitted to NCBI (accession number KY471550). The pure culture is submitted to NCIM, Pune. Fewer studies have been done on strain improvement of the local flora. As the species was isolated from fresh water local flora it was important to subject it for further investigation. When it was cultured on BBM it was clear that it can be grown in laboratory conditions but commercial applications would be limited. So it was important to subject it for further growth studies for better biomass and lipid enhancement. When it comes to the question of optimization it can be done through strain improvement by genetic Engineering studies, mutagenesis and by changing nutrition. In the current study Plackett-Burmann design is been adopted to know the effect of $\mathrm{NaNO}_{3}, \mathrm{CaCl}_{2}$, $\mathrm{MgSO}_{4}, \mathrm{~K}_{2} \mathrm{HPO}_{4}, \mathrm{KH}_{2} \mathrm{PO}_{4}$, Urea and 2-4,D. The advantage of Plackett-Burmann design, it is helpful in choosing all the variables at a time instead of studying them individually (Yang et al., 2014, Mostafa et al., 2016). Plackett-Burmann also helps in finding out the effectiveness of each variable and which further can be used for optimization parameters. Out of seven variables except $\mathrm{K}_{2} \mathrm{HPO}_{4}$ all had positive effect on biomass. $\mathrm{NaNO}_{3}, \mathrm{CaCl}_{2}, \mathrm{MgSO}_{4}, \mathrm{KH}_{2} \mathrm{PO}_{4}$ and Urea had positive effect on lipid. It is critical to have balance between both biomass and lipid in same the media. An effort will be made to optimize both through Response Surface methodology using Box-Behnken design which will be future scope the work.

\section{Materials and Methods}

\section{Collection of water samples}

Different fresh water samples were collected from various water sources such as ponds (from Badami, Bagalkot district, Karnataka, India), domestic water and other resources from Bagalkot district for isolation of microalgae. Collection of fresh water sample was done in clean and dry sterilized water bottles. Water was collected in bottles, which was then sealed by cap and taken to the laboratory.

\section{Isolation of microalgae using BBM}

The collected fresh water samples were inoculated in Bold's Basal Media $\left(\mathrm{NaNO}_{3} 25.0, \mathrm{CaCl}_{2} .2 \mathrm{H}_{2} \mathrm{O} 2.5, \mathrm{MgSO}_{4} .7 \mathrm{H}_{2} \mathrm{O}\right.$ $\begin{array}{lllll}7.5, & \mathrm{~K}_{2} \mathrm{HPO}_{4} 7.5, \quad \mathrm{KH}_{2} \mathrm{PO}_{4} 17.5, & \mathrm{NaCl} & 2.5\end{array}$ EDTA-50.0 KOH 31.0g, $\mathrm{FeSO}_{4} .7 \mathrm{H}_{2} \mathrm{O} 4.98$ $\mathrm{H}_{2} \mathrm{SO}_{4} \quad 1.0 \mathrm{ml}, \quad \mathrm{H}_{3} \mathrm{BO}_{3} 11.42 \quad \mathrm{~g} \quad \mathrm{~L}^{-1}$ Micronutrients- $\quad \mathrm{ZnSO}_{4} .7 \mathrm{H}_{2} \mathrm{O} \quad 8.82$, $\mathrm{MnCl}_{2} .4 \mathrm{H}_{2} \mathrm{O} 1.44, \mathrm{MoO}_{3} 0.71, \mathrm{CuSO} 4.5 \mathrm{H}_{2} \mathrm{O}$ 1.57, $\left.\mathrm{Co}\left(\mathrm{NO}_{3}\right) 2.6 \mathrm{H}_{2} \mathrm{O} 0.49 \mathrm{gL}^{-1}\right)$ for the growth of microalgae present in the water sample. The inoculated media was incubated at room temperature $25^{\circ} \pm 3 \mathrm{C}$ with $\mathrm{pH}$ of 6.8 7.0 . 
To approximately $900 \mathrm{~mL}$ of distilled water were added to all the components in the order specified. Total volume was brought to $1 \mathrm{~L}$ with distilled water. Then covered and autoclaved at $121^{\circ} \mathrm{C}$ for $15 \mathrm{~min}$ at $15 \mathrm{psi}$.

\section{Pure culture of algae (Centrifuge Washing Technique)}

The tubes containing the culture sample was centrifuged at $3000 \mathrm{rpm}$ for 15 minutes. After centrifugation the supernatant was discarded and the cells were suspended in fresh sterile water in tube using vortex mixer to complete centrifuge-washing process. Centrifugation and washing was repeated for three times to expel most of the microorganisms present in the algal sample and the cells were then streaked on the agar plates.

\section{Axenic culture by Streak Plate Technique}

Washed microalgae were streaked on solidified Bold's Basal Medium in asceptic condition and kept for fifteen days for the growth. Repeated streak-platings was carried out to pick up single colony from earlier streaked plates and to make it free from bacteria. From last streaked plates, the single colonies were picked up by loop and allowed to grow in tubes and vials.

Axenic cultures were isolated by repeated subculturing. (Parvin et al., 2007)

\section{Characterization of isolated microalgae}

The isolated microalgae were characterized by observing the cells under light microscope. To a clean and dry glass slide a drop of culture was added. This was covered with cover slip and was observed under $40 \mathrm{X}$ objective. Later pictures were captured in camera attached trinocular microscope (OLYMPUS, India) under 100X. The size of the Chlorella species was measured using stage-ocular micrometer.

\section{Study of growth pattern of Chlorella species}

The isolated Chlorella sp. was grown in $500 \mathrm{ml}$ flask (Borosil) with $250 \mathrm{ml}$ Bold's Basal media with 16:8 hours light and dark cycles at room temperature $25 \pm 2^{0} \mathrm{C}$. The growth pattern of isolated Chlorella species was studied using optical density method. The OD was measured at $540 \mathrm{~nm}$ using spectrophotometer at an interval of 24 hours in triplicates for 30 days.

\section{DNA extraction, PCR amplification and sequencing}

DNA was isolated from axenic culture using genomic DNA isolation kit (Himedia, India). The purity of the DNA was checked by measuring 260/280 ratio and was quantified based on Agarose gel electrophoresis and bands were observed under gel documentation using gel doc (UVCI-1100-MSmajor Science). The extracted DNA was further subjected to PCR amplification using Oligonucleotide primers (18S F: 5'GTAGTCATAKGCTNGTCTS-3'; 18S R 5' GARACCTDGTTAVGACTY-3') (Indhumati et al., 2013, Franziska et al., 2014).

\section{PCR amplification conditions}

PCR reaction was performed in a thermal cycler (MJ research PTC200). The reaction mixture was DNA $1 \mu \mathrm{l}$ (100ng), Forward Primer400ng,Reverse Primer 400ng,dNTPs (10mM each), $4 \mu \mathrm{l}$ 10X Chrom Taq DNA Polymerase, Assay Buffer $10 \mu 1$,Chrom Taq DNA Polymerase Enzyme $(3 \mathrm{U} / \mu \mathrm{l}) 1 \mu \mathrm{l}$, Water $\mathrm{X} \mu \mathrm{l}$, Total reaction volume: $100 \mu \mathrm{l}$

PCR Conditions: (25 cycles): Initial Denaturation: $96^{\circ} \mathrm{C}$ for $1 \mathrm{~min}$, Denaturation: $96^{\circ} \mathrm{C}$ for $10 \mathrm{sec}$, Hybridization: $50{ }^{\circ} \mathrm{C}$ for $5 \mathrm{sec}$ Elongation: $60{ }^{\circ} \mathrm{C}$ for $4 \mathrm{~m}$ (Manisha et al., 2014, Franziska et al., 2014). The amplified PCR was sequenced (Chromous Biotech Pvt., Ltd., Bengaluru, India). 


\section{Phylogenetic analysis}

The 18S rRNA sequence of the strain was searched for homology in NCBI against BLAST. The partial 18S rRNA sequences of the isolated culture showing homology with known sequences of Chlorella sp. KMMCC FC-69 18S (Accession No. GQ122376.1) Chlorella sorokiniana strain UTEX 280518 S (Accession No KR092112.1) existing in the database.

\section{Plackett-Burman design}

Seven variables were selected viz., $\mathrm{NaNO}_{3}$, $\mathrm{CaCl}_{2}, \mathrm{MgSO}_{4}, \mathrm{~K}_{2} \mathrm{HPO}_{4}, \mathrm{KH}_{2} \mathrm{PO}_{4}$, Urea, 24,D. Each variable had two levels: '-' low level and ' + ' high level, all were chosen in $g$ $\mathrm{L}^{-1}$ and 2,4-D in $\mathrm{mg} \mathrm{L}^{-1}$. Higher concentration was selected as twice the higher concentration of BBM composition and lower concentration was half of the BBM composition. Urea and 2,4-D were chosen as an additional variables apart from the BBM composition. Urea a synthetic fertilizer, major source of nitrogen for the plant nutrition is a widely used in agriculture. The surface runoff water of agricultural land consists of urea which leads to Eutrophication problem in water bodies.

2,4-D at lower concentration is used as a growth hormone and at higher concentration used as a herbicide and is also one of the component of agricultural surface runoff water. Algae can be used for bioremediation of waste water bodies and also the biomass obtained from algal blooms can be used as a source of biofuels. The isolated species was subjected for the effect of urea and 2,4-D to reveal their effect on biomass and lipid content. Rest of the media composition was unchanged and was kept as dummy variables (Devendra and Pravin 2010, Reddy and Rao 2012, Manisha et al., 2014, Yang et al., 2014, Sheekh et al., 2015). The layout of PlackettBurmann is shown in table 1.

\section{Biomass study}

The biomass was determined based on the cell density using Neabauer haemocytometer for every interval 48 hours (Weena et al., Larson and Aburg, 1997). The data was collected in triplicates.

\section{Lipid estimation}

Lipid was photo metrically estimated on seventh day of inoculation using colorimeter at $430 \mathrm{~nm} .5 \mathrm{ml}$ algal sample from each run was centrifuged and the fresh pellet was used for quantification and was estimated in terms of $\mathrm{mgL}^{-1}$ of the media. The quantification is based on the fatty acids dissolved in chloroform. Saponified fatty acids were mixed in a copper reagent and chloroform was added and was measured colorimetrically by using sodium diethyl thiocarbamate which develops into yellow color and is proportional to the amount of lipid present in it. The palmitic acid was used for standard curve (make Himedia, 99\% purity) (Duncombe, 1963; Wawrik and Harriman, 2010).

\section{Results and Discussion}

In most of the water samples green algae were found viz., Chlorella sp., filamentous algae and diatoms. Chlorella sp. was isolated and further subjected for pure culture.

\section{Isolation, characterization and growth of microalgae}

Among different samples, Filamentous algae, Diatoms and Microalgae were found. Chlorella was chosen because of its wide adaptability over wide range of $\mathrm{pH}$. Based on the microscopic observation, cells of Chlorella are round, small and normally individually dissociated (Ying et al., 2009 and Rosen 1990). The size of the Chlorella sp., ranged from $6-12 \mu \mathrm{m}$ using stage-ocular micrometer. 
Table.1 Seven variables with twelve runs of two level Plackett-Burmann design

\begin{tabular}{|c|c|c|c|c|c|c|c|}
\hline Runs & $\mathrm{NaNO}_{3}$ & $\mathrm{CaCl}_{2}$, & $\mathrm{MgSO}_{4}$ & $\mathrm{~K}_{2} \mathrm{HPO}_{4}$ & $\mathrm{KH}_{2} \mathrm{PO}_{4}$ & \multirow{2}{*}{$\begin{array}{l}\text { Urea } \\
\mathrm{g} \mathrm{L}^{-1}\end{array}$} & \multirow{2}{*}{$\begin{array}{l}\text { 2-4,D } \\
\mathrm{mg} \mathrm{L}^{-1}\end{array}$} \\
\hline & \multicolumn{5}{|c|}{$\mathrm{g} \mathrm{L}^{-1}$ stock solution } & & \\
\hline+ & 50 & 5 & 15 & 15 & 35 & 5 & 3 \\
\hline- & 12.5 & 1.2 & 3.2 & 3.25 & 8.75 & 0.1 & 0.1 \\
\hline 1 & + & - & + & - & - & - & + \\
\hline 2 & - & - & - & + & + & + & - \\
\hline 3 & + & - & + & + & - & + & - \\
\hline 4 & - & + & - & - & - & + & + \\
\hline 5 & - & - & + & + & + & - & + \\
\hline 6 & - & + & + & - & + & - & - \\
\hline 7 & - & - & - & - & - & - & - \\
\hline 8 & - & + & + & + & - & + & + \\
\hline 9 & + & + & - & + & + & - & + \\
\hline 10 & + & + & + & - & + & + & - \\
\hline 11 & + & - & - & - & + & + & + \\
\hline 12 & + & + & - & + & - & - & - \\
\hline
\end{tabular}

'+' level indicates high value and '-' level indicates low value.

Table.2 The Estimated effects and coefficients for biomass response of Plackett-Burman

\begin{tabular}{|l|l|l|l|l|l|}
\hline Variable & Effect & Co-efficient & SE Coefficient & t-value & p-value \\
\hline Constant & & 13.7917 & 0.5715 & 24.13 & 0.000 \\
\hline $\mathrm{NaNO}_{3}$ & 0.2833 & 0.1417 & 0.5715 & 0.25 & 0.816 \\
\hline $\mathrm{CaCl}_{2}$ & 2.8833 & 1.4417 & 0.5715 & 2.52 & $0.065^{*}$ \\
\hline $\mathrm{MgSO}_{4}$ & 1.0833 & 0.5417 & 0.5715 & 0.95 & 0.397 \\
\hline $\mathrm{K}_{2} \mathrm{HPO}_{4}$ & -0.3833 & -0.1917 & 0.5715 & -0.34 & 0.754 \\
\hline $\mathrm{KH}_{2} \mathrm{PO}_{4}$ & 0.0167 & 0.0083 & 0.5715 & 0.01 & 0.989 \\
\hline Urea & 7.6833 & 3.8417 & 0.5715 & 6.72 & $0.003 *$ \\
\hline 2-4,D & 1.5500 & 0.7750 & 0.5715 & 1.36 & 0.247 \\
\hline
\end{tabular}

SE, standard error; *Significant at $95 \%$ level $(p<0.05)$

Table.3 ANOVA of variance for Biomass response

\begin{tabular}{|l|l|l|l|l|l|l|}
\hline Source & DF & Seq SS & Adj SS & Adj MS & F value & P value \\
\hline Main Effects & 7 & 213.45 & 213.45 & 30.493 & 7.78 & 0.033 \\
\hline Residual Error & 4 & 15.68 & 15.68 & 3.919 & & \\
\hline Total & 11 & 229.13 & & & & \\
\hline
\end{tabular}


Table.4 The Estimated effects and coefficients for lipid response of Plackett-Burman

\begin{tabular}{|l|l|l|l|l|l|}
\hline Variable & Effect & Co-efficient & SE Coefficient & t-value & p-value \\
\hline Constant & & 71.750 & 6.158 & 11.65 & 0.000 \\
\hline $\mathrm{NaNO}_{3}$ & 16.833 & 8.417 & 6.158 & 1.37 & 0.243 \\
\hline $\mathrm{CaCl}_{2}$ & -2.500 & -1.250 & 6.158 & -0.20 & 0.849 \\
\hline $\mathrm{MgSO}_{4}$ & 14.500 & 7.250 & 6.158 & 1.18 & 0.304 \\
\hline $\mathrm{K}_{2} \mathrm{HPO}_{4}$ & -10.167 & -5.083 & 6.158 & -0.83 & 0.455 \\
\hline $\mathrm{KH}_{2} \mathrm{PO}_{4}$ & 14.167 & 7.083 & 6.158 & 1.15 & 0.314 \\
\hline Urea & 9.167 & 4.583 & 6.158 & 0.74 & 0.498 \\
\hline 2-4,D & -8.167 & -4.083 & 6.158 & -0.66 & 0.544 \\
\hline
\end{tabular}

SE, standard error; $(p<0.05)$

Table.5 ANOVA of variance for lipid response

\begin{tabular}{|l|l|l|l|l|l|l|}
\hline Source & DF & Seq SS & Adj SS & Adj MS & F value & P value \\
\hline \begin{tabular}{l} 
Main Effects \\
\hline $\begin{array}{l}\text { Residual } \\
\text { Error }\end{array}$
\end{tabular} & 7 & 2864 & 2864 & 409.1 & 0.90 & 0.578 \\
\hline Total & 11 & 1820 & 1820 & 455.1 & & \\
\hline
\end{tabular}

Fig.1 Microscopic view of Chlorella sorokiniana PCPBEC under 100x compound microscope

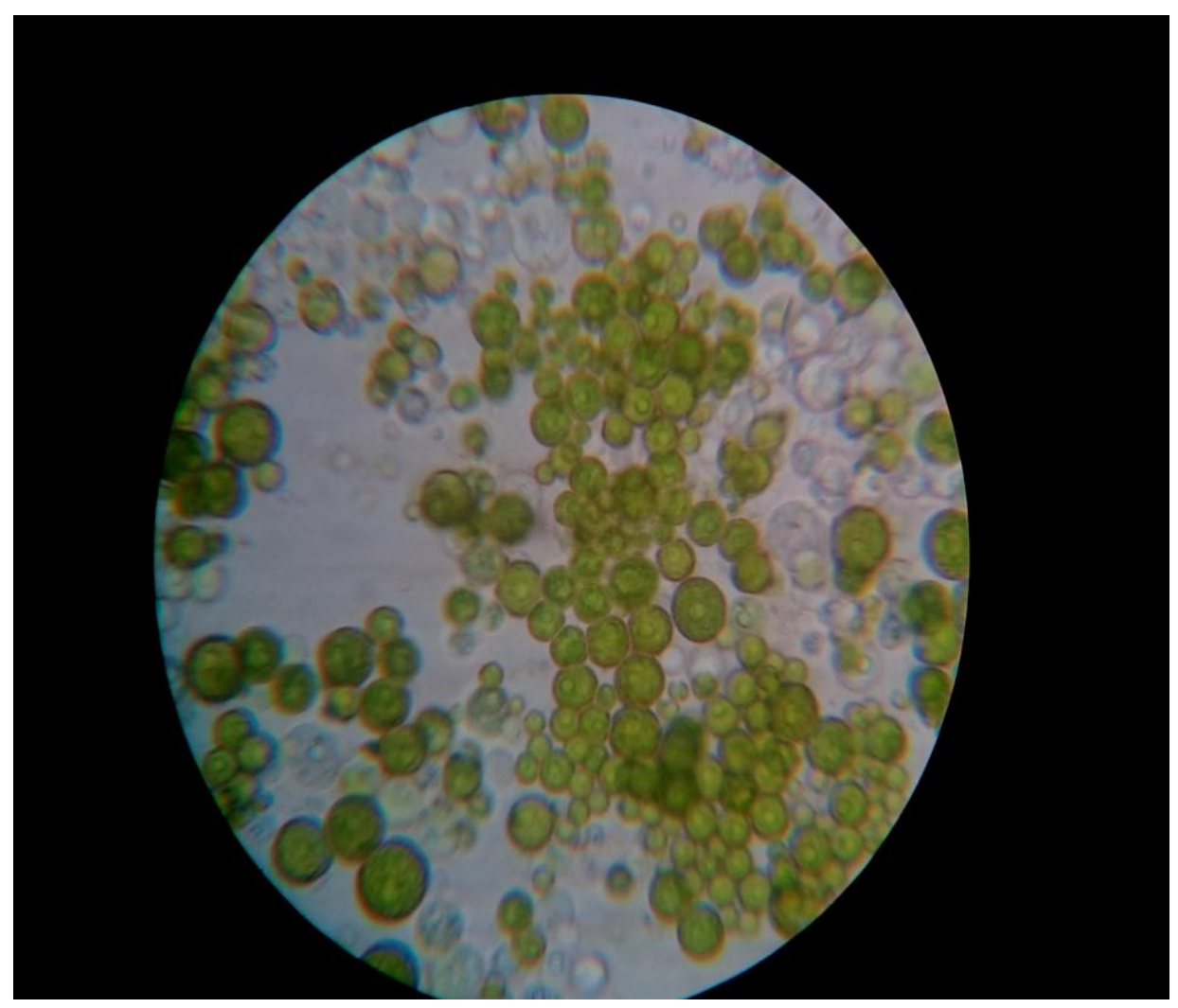


Fig.2 Extraction of genomic DNA from Isolated sample

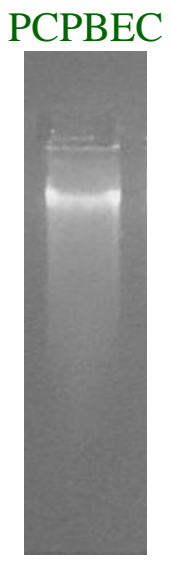

Fig.3 PCR amplification of 18s rDNA region from the isolate. The size of PCR amplified product is $\sim 2 \mathrm{~kb}$ (sequences)

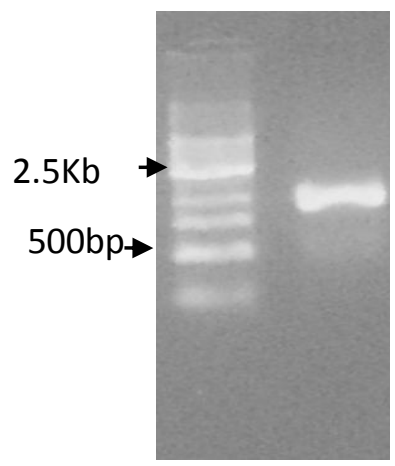

Fig.4 Results of blast analysis of Chlorella sorokiniana PCPBEC compared with other related species

\begin{tabular}{|c|c|c|c|c|c|}
\hline 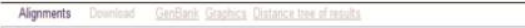 & & & & & \\
\hline Description & $\begin{array}{l}\text { Mex } \\
\text { score }\end{array}$ & $\begin{array}{l}\text { Total Qvery } \\
\text { score cover }\end{array}$ & E & Itent & Accessicen \\
\hline 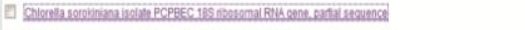 & 3012 & $3072100 \%$ & 00 & $100 \%$ & $\$ 47715001$ \\
\hline 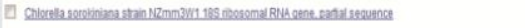 & 3049 & 3049996 & 00 & 99\% & mosemesu \\
\hline 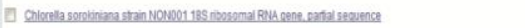 & 3049 & $3049100 \%$ & 00 & $99 \%$ & WF101221.1. \\
\hline 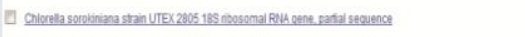 & 3049 & $3049100 \%$ & 00 & $99 \%$ & KRQ⿻日2:121 \\
\hline 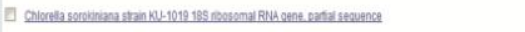 & 3049 & $3049100 \%$ & 00 & 996 & KF 444071 \\
\hline 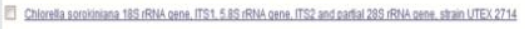 & 3049 & $3049100 \%$ & 00 & $99 \%$ & Le212421 \\
\hline 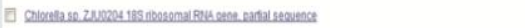 & 3049 & $3049100 \%$ & 00 & $99 \%$ & 15090561 \\
\hline 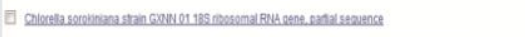 & 3049 & $3049100 \%$ & 00 & $99 \%$ & Eusnesers: \\
\hline 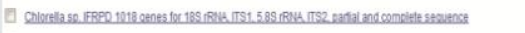 & 3049 & $3049100 \%$ & 00 & $99 \%$ & 18280909891 \\
\hline 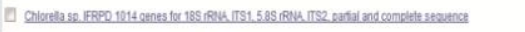 & 3049 & $3049100 \%$ & 00 & 996 & 482506971 \\
\hline 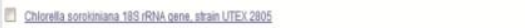 & 3009 & $3049100 \%$ & 00 & 996\% & 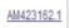 \\
\hline 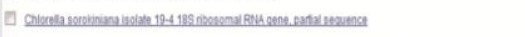 & 304 & $3044100 \%$ & 00 & 996 & 129agere 1 \\
\hline 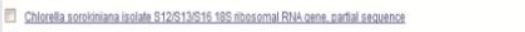 & 304 & $3044100 \%$ & 00 & $99 \%$ & KTE52929:1 \\
\hline 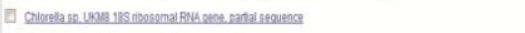 & 3044 & $3044100 \%$ & 00 & $99 \%$ & kTL50921 \\
\hline 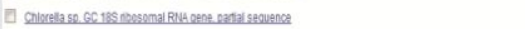 & 304 & $3044100 \%$ & 00 & $99 \%$ & KETI37421 \\
\hline 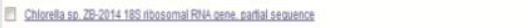 & 3044 & $304499 \%$ & 00 & $99 \%$ & kn27248991. \\
\hline 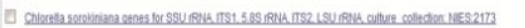 & 304 & $3044100 \%$ & 00 & $99 \%$ & $\triangle 863102021$ \\
\hline
\end{tabular}


Fig.5 Phylogenetic Analysis of Chlorella sorkiniana PCPBEC accession number KY471550 to predict the evolutionary relationship
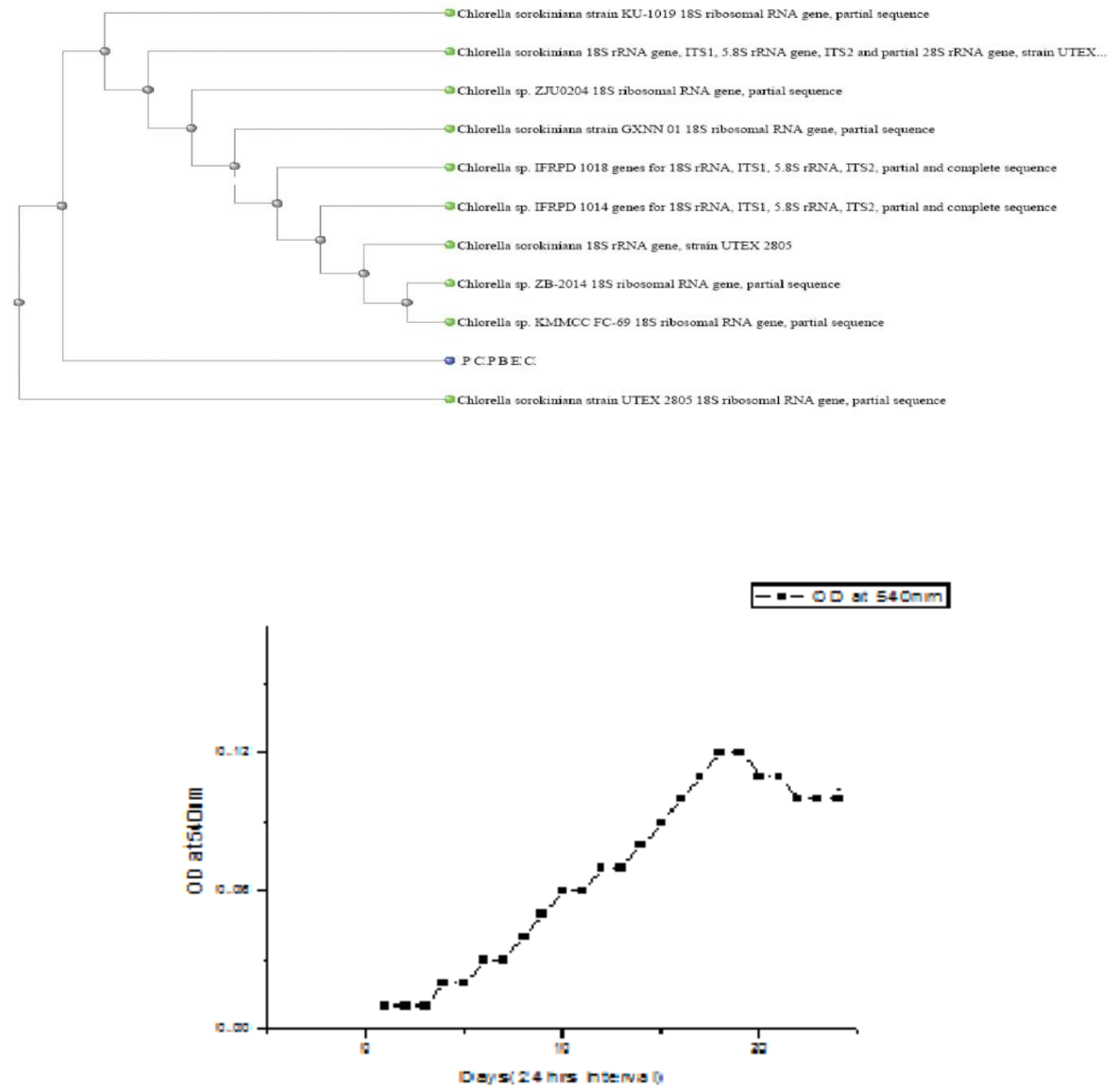

Graph 1. Growth pattern of Chlorella sp.

Chlorella sorokiniana observed under 100x compound microscope (Fig. 1).

\section{Study of growth pattern of Chlorella species}

The growth pattern of isolated Chlorella species was studied using optical density method. The OD was measured at $540 \mathrm{~nm}$ using colorimeter at an interval of $24 \mathrm{hrs}$.

The growth pattern is shown in the graph1. (Graph was plotted using Orion-8).
DNA extraction, PCR amplification and sequencing

The blast sequences of Chlorella sorokiniana PCPBEC:

CTGACTTTATACTGGTGAAACTGCGAA TGGCTCATTAAATCAGTTATAGTTTAT TTGATGGTACCTACTACTCGGATACCC GTAGTAAATCTAGAGCTAATACGTGCG TAAATCCCGACTTCTGGAAGGGACGTA TTTATTAGATAAAAGGCCGACCGGGCT CTGCCCGACTCGCGGTGAATCATGATA 
ACTTCACGAATCGCATGGCCTTGCGCC GGCGATGTTTCATTCAAATTTCTGCCC TATCAACTTTCGATGGTAGGATAGAGG CCTACCATGGTGGTAACGGGTGACGG AGGATTAGGGTTCGATTCCGGAGAGG GAGCCTGAGAAACGGCTACCACATCC AAGGAAGGCAGCAGGCGCGCAAATTA CCCAATCCTGACACAGGGAGGTAGTG ACAATAAATAACAATACTGGGCCTTTT CAGGTCTGGTAATTGGAATGAGTACAA TCTAAACCCCTTAACGAGGATCAATTG GAGGGCAAGTCTGGTGCCAGCAGCCG CGGTAATTCCAGCTCCAATAGCGTATA TTTAAGTTGCTGCAGTTAAAAAGCTCG TAGTTGGATTTCGGGTGGGGCCTGCCG GTCCGCCGTTTCGGTGTGCACTGGCAG GGCCCACCTTGTTGCCGGGGACGGGCT CCTGGGCTTCACTGTCCGGGACTCGGA GTCGGCGCTGTTACTTTGAGTAAATTA GAGTGTTCAAAGCAGGCCTACGCTCTG AATACATTAGCATGGAATAACACGAT AGGACTCTGGCCTATCCTGTTGGTCTG TAGGACCGGAGTAATGATTAAGAGGG ACAGTCGGGGGCATTCGTATTTCATTG TCAGAGGTGAAATTCTTGGATTTATGA AAGACGAACTACTGCGAAAGCATTTG CCAAGGATGTTTTCATTAATCAAGAAC GAAAGTTGGGGGCTCGAAGACGATTA GATACCGCTGACTTTATACTGGTGAAA CTGCGAATGGCTCATTAAATCAGTTAT AGTTTATTTGATGGTACCTACTACTCG GATACCCGTAGTAAATCTAGAGCTAAT ACGTGCGTAAATCCCGACTTCTGGAAG GGACGTATTTATTAGATAAAAGGCCGA CCGGGCTCTGCCCGACTCGCGGTGAAT CATGATAACTTCACGAATCGCATGGCC TTGCGCCGGCGATGTTTCATTCAAATT TCTGCCCTATCAACTTTCGATGGTAGG ATAGAGGCCTACCATGGTGGTAACGG GTGACGGAGGATTAGGGTTCGATTCCG GAGAGGGAGCCTGAGAAACGGCTACC ACATCCAAGGAAGGCAGCAGGCGCGC AAATTACCCAATCCTGACACAGGGAG GTAGTGACAATAAATAACAATACTGG GCCTTTTCAGGTCTGGTAATTGGAATG AGTACAATCTAAACCCCTTAACGAGGA
TCAATTGGAGGGCAAGTCTGGTGCCAG CAGCCGCGGTAATTCCAGCTCCAATAG CGTATATTTAAGTTGCTGCAGTTAAAA AGCTCGTAGTTGGATTTCGGGTGGGGC CTGCCGGTCCGCCGTTTCGGTGTGCAC TGGCAGGGCCCACCTTGTTGCCGGGGA CGGGCTCCTGGGCTTCACTGTCCGGGA CTCGGAGTCGGCGCTGTTACTTTGAGT AAATTAGAGTGTTCAAAGCAGGCCTAC GCTCTGAATACATTAGCATGGAATAAC ACGATAGGACTCTGGCCTATCCTGTTG GTCTGTAGGACCGGAGTAATGATTAAG AGGGACAGTCGGGGGCATTCGTATTTC ATTGTCAGAGGTGAAATTCTTGGATTT ATGAAAGACGAACTACTGCGAAAGCA TTTGCCAAGGATGTTTTCATTAATCAA GAACGAAAGTTGGGGGCTCGAAGACG ATTAGATACCGTCCTAGTCTCAACCAT AAACGATGCCGACTAGGGATCGGCGG ATGTTTCTTCGATGACTCCGCCGGCAC CTTATGAGAAATCAAAGTTTTTGGGTT CCGGGGGGAGTATGGTCGCAAGGCTG AAACTTAAAGGAATTGACGGAAGGGC ACCACCAGGCGTGGAGCCTGCGGCTTA ATTTGACTCAACACGGGAAAACTTACC AGGTCCAGACATAGTGAGGATTGACA GATTGAGAGCTCTTTCTTGATTCTATG GGTGGTGGTGCATGGCCGTTCTTAGTT GGTGGGTTGCCTTGTCAGGTTGATTCC GGTAACGAACGAGACCTCAGCCTGCT AAATAGTCACGGTTGGCTCGCCAGCCG GCGGACTTCTTAGAGGGACTATTGGCG ACTAGCCAATGGAAGCATGAGGCAAT AACAGGTCTGTGATGCCCTTAGATGTT CTGGGCCGCACGCGCGCTACACTGATG CATTCAACGAGCCTAGCCTTGGCCGAG AGGCCCGGGTAATCTTTGAAACTGCAT CGTGATGGGGATAGATTATTGCAATTA TTAATCTTCAACGAGGAATGCCTAGTA AGCGCAAGTCATCAGCTTGCGTTGATT ACGTCCCTGCCCTTTGTACACACCGCC CGTCGCTCCTACCGATTGGGTGTGCTG GTGAAGTGTTCGGATTGGCGACCGGG GGCGGTCTCCGCTCTCGGCCGCCGAGA AGTCATAAAC 


\section{Phylogenetic analysis}

\section{Plackett- Burman design}

The experimental design analysis was done using MINITAB-14 software. To test the significance of the hypothesis $p$-value was used. If the p-value was less than 5\%, indicates the significance of the chosen variable and greater value indicates nonsignificance of the hypothesis. Among the seven variables chosen for biomass urea and $\mathrm{CaCl}_{2}$ had positive effect with p-value 0.003 and 0.685 , indicating highest effect on biomass. Rest of the variables had positive effect except $\mathrm{K}_{2} \mathrm{HPO}_{4}$. But urea had a highest effect among seven variables. In fact the urea had a greater effect than the $\mathrm{NaNO}_{3}$ indicating the species can be further explored for Agricultural surface runoff water treatment. In contrast to this, for lipid studies the effect of these variables was different. The p-value was 0.578 indicating non significance. $\mathrm{CaCl}_{2}$, $\mathrm{K}_{2} \mathrm{HPO}_{4}$ and $2-4 \mathrm{D}$, had negative effect. Interestingly $\mathrm{NaNO}_{3}$ had highest positive effect followed by $\mathrm{MgSO}_{4}, \mathrm{KH}_{2} \mathrm{PO}_{4}$ and Urea. Many reports suggest that increased nitrogen concentration reduces lipid production but that was not observed in this study. In fact Urea had a positive effect on both biomass and lipid. This clearly indicates that further urea need to be properly evaluated for standardizing both biomass and lipid production. The results are shown in Table 2, 3, 4 and 5 (Fig. 1-5).

The current study aimed at isolating the local flora and screening it for biomass and lipid enhancement studies. Effort was made to culture and improve the strain for its for commercial applications. Morphological and molecular characterization was done through gene sequencing, which revealed the species to be microalgae of genera Chlorella sorokiniana PCPBEC (accession number KY471550) and database is been submitted to NCBI. Further it was subjected to Biomass and lipid enhancement studies through Plackett-Burmann, additionally to $\mathrm{BBM}$ media. $\mathrm{NaNO}_{3} \quad \mathrm{CaCl}_{2}, \mathrm{MgSO}_{4}, \quad \mathrm{~K}_{2} \mathrm{HPO}_{4}$, $\mathrm{KH}_{2} \mathrm{PO}_{4}$, Urea, and 2-4,D (Urea and 2,4-D were added deliberately, which are pollutants found in agriculture surface runoff water). Biomass had a better response for all the variables except $\mathrm{K}_{2} \mathrm{HPO}_{4}$. Variables $\mathrm{CaCl}_{2}$ $\mathrm{K}_{2} \mathrm{HPO}_{4}, 2-4$,D had a negative effect on lipid enhancement. Overall $\mathrm{K}_{2} \mathrm{HPO}_{4}$ had a negative effect on both studies. So Urea had resulted positive effects on both studies which need to be further optimized. The study has a scope for further media optimization. The isolate can be used for waste water treatment studies.

\section{Acknowledgments}

The research was funded by TEQIP-II (Technical Education Quality Improvement Programme- Phase II) a World Bank initiation, as seed money for young scientist.

\section{References}

Barry H., Rosen, (1990), Microalgae Identification for Aquaculture, Florida Aquafarms.

Bischoff, H.W., and Bold, H.C., (1963). Phycological studies IV. Some soil algae from Enchanted Rock and related algal species, University of Texas Publication 6318: [1]-95.

Boris Wawrik., and Biran H., Harriman, 2010, Rapid colorimetric quantification of lipid algal culture, Journal of microbiological methods, 24: 156-161.

Box G.E.P., and Behnken D.W., (1960)., Simplex-Sum Designs: A Class of Second Order Rotatable Designs Derivable From Those of First Order, The Annals of Mathematical Statistics, 31(4): 838-864

Chisti Yusuf, (2007), Biodiesel from microalgae, Biotechnol advances, 25:294-306. 
Chisti Yusuf, (2008), Do biofuels from microalgae beat biofuels from terrestrial plants?, Trends Biotechnology, 26:351352.

Devendra V. Deshmukh and Pravin R. Puranik, (2010), Application of Plackett-Burman Design to evaluate Media Components Affecting Antibacterial Activity of Alkaliphilic Cyanobacteria Isolated from Lonar Lake, Turkish Journal of Biochemistry, 35 (2); 114-120.

Duncombe W., G, (1963), The colorimetric Micro-determination of long chain fatty acids, Biochemistry journal, 88: 7-11

Fangfang Yang., Lijuan Long, Xiumei Sun, Hualian $\mathrm{Wu}$, Tao $\mathrm{Li}$ and Wenzhou Xiang, (2014), Optimization of medium using response surface methodology for lipid production by Scenedesmus sp., Marine Drugs, 12: 1245-1257.

Franziska Bleeke., Vincent M. Rwehumbiza., Dominik Winckelmann and Gerd Klock, 2014, Isolation and Characterization of New Temperature Tolerant Microalgal Strains for Biomass Production, Energies, 7:7847-7856

Indhumati Ponnaswamy., Soundararajan Madhavan and Syed Sahabudeen, 2013, Isolation and Characterization of Green Microalgae for Carbon Sequestration, Waste Water Treatment and Bio-fuel Production, International Journal of Bio-Science and Bio-Technology, 5(2): 17-25

Manisha Tale., Sukhendhu Ghosh, Balasaheb Kapadnis and Sharad Kale, 2014,
Isolation and characterization of microalgae for biodiesel production from Nisargruna biogas plant effluent, Bioresource Technology, 169:328-335

Michael Hannon., Javier Gimpel., Miller Tran, Beth Rasala and Stephen Mayfield.,(2010) Biofuels from microalgae: challenges and potential, Biofuels, 5:763-784

Mostafa M., El-Sheekh, Hanan M., Khairy, Saly F., Gheda and Rania A., ElShenody, (2016), Application of Plackett-Burman design for the high production of some valuable metabolites in marine alga Nannochloropsis oculata, Egyptian Journal of Aquatic Research,42:57-64

Parvin M., Zannat M.,N.., and Habib M.A.B., (2007), Two Important Techniques for Isolation of Microalgae, Asian Fisheries Science, 20:117-124.

Plackett R. L. and Burman J. P., (1946), "The design of optimum multifactorial experiments", Biometrica, 33:305-325.

Sivasubramanian V., (2009), Current status of Research on algal bio-fuels in India, Journal of Algal Biomass Utilization, 1(1): 1-8.

Venkata Ramana Reddy M. B., Rao C. S. and Lakshmana Rao S. S., (2012), Evaluation of Process Parameters and Media Components by Plackett-Burman Design for Enhancement of Biomass using Cyanobacteria (Anabaena ambigua), International Journal of ChemTech Research, 4(2):761-766.

\section{How to cite this article:}

Premjyoti C. Patil and Bharati S. Meti. 2018. Isolation of Microalgae for Biomass and Lipid Enhancement through Plackett-Burmann Design. Int.J.Curr.Microbiol.App.Sci. 7(03): 25082518. doi: https://doi.org/10.20546/ijcmas.2018.703.290 